\title{
ESTUDO COMPARATIVO DO VALOR NUTRICIONAL DAS ÁGUAS COM SABOR DISPONÍVEIS EM PORTUGAL
}

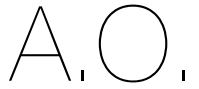
ARTIGO ORIGINAL

1 Escola Superior de Tecnologia da Saúde de Coimbra do Instituto Politécnico de Coimbra Rua 5 de Outubro, 3046-854 Coimbra Portugal

`Endereço para correspondência:

Marta Laranjeiro Pinto Escola Superior de Tecnologia da Saúde de Coimbra, Rua 5 de Outubro, 3046-854 Coimbra, Portuga pinto.mlar@gmail.com

Histórico do artigo:

Recebido a 5 de maio de 2020 Aceite a 30 de junho de 2020

\section{COMPARATIVE STUDY OF THE NUTRITIONAL VALUE OF THE FLAVORED WATERS COMMERCIALIZED IN PORTUGAL}

Marta Laranjeiro Pinto" ${ }^{1 *}$; Ana Lúcia Baltazar ${ }^{1}$

RESUMO

INTRODUÇÃo: Nos últimos anos, aumentou a oferta de águas com sabor, vulgarmente apelidadas de águas aromatizadas, disponíveis em Portugal. Também o seu consumo sofreu um aumento significativo.

OBJETIVOS: Comparar a composição e valor nutricional de águas com sabores produzidas por cinco marcas diferentes.

METODOLOGIA: Consulta online de rótulos de águas com sabores disponíveis em Portugal, com e sem gás.

RESULTADOS: A sua composição varia entre a combinação de água, entre 82 e 98\%, e de sumo, entre 0 e 12\%. Para além do ácido cítrico, presente em todas as marcas, os aditivos alimentares mais usados são os adoçantes (nutritivos e não-nutritivos) e os conservantes. Estas diferenças refletem-se no valor nutricional de cada água. As maiores diferenças estão relacionadas com o valor energético, o qual varia entre quatro e $26 \mathrm{Kcal} / 100 \mathrm{~mL}$, e com o teor de açúcares, entre 0 e 5,3 g/100 mL. Por outro lado, algumas águas possuem um valor considerável de sal, o qual pode ascender a 0,17 g/100 mL.

CONCLUSÕES: Como conclusão, as águas com sabor são refrigerantes que contêm muitos aditivos podendo o seu consumo contribuir para um aumento significativo do aporte energético e de açúcar diário. Por este motivo devem ser consumidas ocasionalmente e nunca como substitutos da água.

\section{PALAVRAS-CHAVE}

Aditivos alimentares, Alimentos processados, Hidratação, Refrigerantes

ABSTRACT

INTRODUCTION: In the last years, there has been an increase in both the flavored waters available in Portugal and in their consumption. OBJECTIVES: Compare the composition and the nutritional value of flavored waters produced by five different brands. METHODOLOGY: Websites from various hypermarkets and specific companies were consulted to access the information regarding ingredients and nutritional value of the analyzed flavored waters.

RESULTS: In general, the percentage of water from different brands varies between 82 and $98 \%$, which is related to the amount of juice used, which goes from 0 to $12 \%$. Besides these two ingredients, flavored waters have many food additives, like citric acid, present in all waters, sweeteners (nutritive or non-nutritive) and preservatives. These different compositions have an impact on their nutritional value. The main differences are related to the energy density, which varies between 4 and $26 \mathrm{Kcal} / 100 \mathrm{~mL}$, and sugars, which vary between 0 and $5.3 \mathrm{~g} / 100 \mathrm{~mL}$. Moreover, some waters have a considerable amount of salt, up to $0.17 \mathrm{~g} / 100 \mathrm{~mL}$.

CoNCLUSIONS: Despite being perceived as an excellent alternative, flavored waters are, in fact, sodas that have many food additives in their composition. Moreover, their consumption may contribute to a significant increase in the daily intake of calories and sugar. For these reasons, flavored waters should be consumed occasionally and never as a substitute for water.

\section{KEYWORDS}

Food additives, Processed food, Hydration, Soda

\section{INTRODUÇÃO}

A água é um alimento essencial à vida, tendo diversas funções incluindo manutenção do equilibrio hidroelectrolítico, termorregulação, transporte e participação em reações nos processos de digestão, absorção, metabolismo e excreção (1). Apesar de alguma variabilidade em relação às recomendações, é globalmente aconselhado um consumo diário de água de $1500 \mathrm{~mL}$, podendo estes valores ser superiores em situações específicas, incluindo doença ou gravidez (2). A Autoridade Europeia para a Segurança dos Alimentos (EFSA), em 2010, recomendava como ingestão adequada 2 litros para as mulheres e 2,5 litros para os homens, incluindo água proveniente de fontes alimentares ou outras bebidas (3). Apesar de ser uma informação conhecida pela generalidade da população, são muitos aqueles que não cumprem estas recomendações. Resultados de um inquérito conduzido, em Portugal, pelo Instituto Nacional de Saúde Doutor Ricardo Jorge (INSA), em 2016, e apresentado nas 
"I Jornadas da Água: uma questão de Saúde Pública", revela que 35\% dos inquiridos bebe diariamente entre 0,5 litros e 1 litro de água, e pouco mais de 30\% consome entre 1 litro e 1,5 litros. Apesar de a distribuição da amostra não ser representativa da população portuguesa em relação ao género e grupo etário, os resultados revelam algumas tendências a nível nacional (4).

Desde há muito tempo que a água mineral gasocarbónica, com gás natural, faz parte do nosso dia a dia. Estudos mostram que este tipo de água é tão eficaz na hidratação como água natural (5), podendo ter alguns benefícios em situações de dispepsia e obstipação (6). Nos últimos anos, temos assistido a um aumento na oferta de águas com sabores no nosso país, mais conhecidas por águas aromatizadas, com ou sem gás, sendo muito usadas por pessoas que dizem não gostar de água. Além disso, o facto de ter água no nome, sugere que se trata de um produto saudável. Mas será mesmo assim? De que são feitas estas "águas" e qual a sua composição nutricional? Neste trabalho efetuou-se uma comparação dos produtos que existem no mercado, nomeadamente a nível de ingredientes e composição nutricional, com o objetivo de tentar perceber se efetivamente se trata de uma boa alternativa à água natural.

\section{METODOLOGIA}

Este trabalho foi elaborado através da consulta online de rótulos de águas aromatizados disponíveis em Portugal, com e sem gás. Foram incluídas águas das quatro principais marcas Portuguesas, de um universo de cinco, e uma de marca branca, que tivesse disponível pelo menos 2 produtos diferentes, para ter uma amostra representativa. Para cada marca foram incluídas entre 2 e 5 águas, em função da oferta e de forma a abranger uma elevada variedade em termos de sabores. Os gráficos foram realizados usando o GraphPad Prism (versão 8.4.2), sendo apresentadas a média e o erro padrão para cada marca.

\section{RESULTADOS}

Ao fazer uma pesquisa à oferta de águas com sabores existentes no mercado português, é possível observar que atualmente existem produtos desenvolvidos por várias empresas portuguesas. A maioria delas são reconhecidas por comercializarem água mineral, mas com a evolução do mercado e a tecnologia associada ao setor alimentar, gradualmente alargaram o espectro de artigos oferecidos.

Este estudo foca-se nas águas desenvolvidas por 5 marcas diferentes, que são identificadas com as letras A, B, C, D e E. No caso específico da marca $\mathrm{C}$, por haver duas gamas de produtos com composições bastante diferentes, são separadas em C1 e C2. Estas águas estão disponíveis em formatos muito diversos, variando entre garrafas de 0,25, 0,33, 0,5, 1 e 2 litros. Uma das marcas disponibiliza ainda uma versão "kids" de 0,2 litros. Em termos de sabores há uma enorme variedade no mercado. Há opções mais simples, como limão, frutos vermelhos ou maracujá, e misturas mais complexas como goiaba e toranja ou maçã e chá branco.

Analisando o rótulo deste tipo de produtos é possível observar que na verdade se designam de refrigerantes, sendo que a percentagem de água varia bastante: aproximadamente $98 \%$ no caso A, $97 \%$ no caso C e $82 \%$ nas águas B (Figura 1a). Para as outras marcas, esta informação não é fornecida no rótulo. No caso específico das marcas A e D, é utilizada água mineral natural gasocarbónica, enquanto que as águas da marca E são gaseificadas artificialmente. As marcas B e C apresentam-se como alternativas sem gás.

Para conferir o sabor, a maior parte das marcas usa a combinação de aromas, naturais ou artificiais, e sumos à base de concentrados, sendo a percentagem de sumo usada muito variada. No caso das marcas $B$ e C2, esta percentagem ronda 12\%. Já no caso das A, C1 e D, varia entre 0,1 e $3 \%$. Especificamente nas águas da marca E, são usados exclusivamente aromas artificiais (Figura 1b).

Outro ingrediente que é usado transversalmente por todas as águas analisadas é ácido cítrico (E330) como regulador de acidez (Tabela 1). Este é o ácido mais usado na indústria alimentar, nomeadamente em produtos em que se pretende intensificar o sabor cítrico. Este produto funciona ainda como um antioxidante indireto, na medida em que funciona como quelante de metais envolvidos em processos de oxidação (7). No caso das águas da marca B, não são utilizados mais nenhuns ingredientes. As outras marcas recorrem ainda a edulcorantes para conferir o sabor doce a estes refrigerantes. No caso das marcas $\mathrm{C} 1$ e D, são usados adoçantes nutritivos (que possuem valor energético), especificamente a frutose ou xarope de glucose-frutose, respetivamente.

No caso da A e E, são usados adoçantes não nutritivos (não possuem valor energético e têm doçura acentuada), nomeadamente sucralose (E955) e/ou acessulfame K (E950). A sucralose provém da modificação da sacarose, tendo um poder adoçante $600 x$ superior a esta, enquanto o acessulfame K é produzido a partir do ácido acético e o seu poder adoçante é cerca de $200 x$ superior à sacarose (8). No caso das águas da marca E, estas possuem também maltodextrina, um polissacarídeo obtido a partir da hidrólise parcial do amido, frequentemente usando com espessante.

\section{Figura 1}

Conteúdo de água (a) e de concentrado de sumo de fruta (b) das águas com sabor em função da marca

a. Conteúdo de água

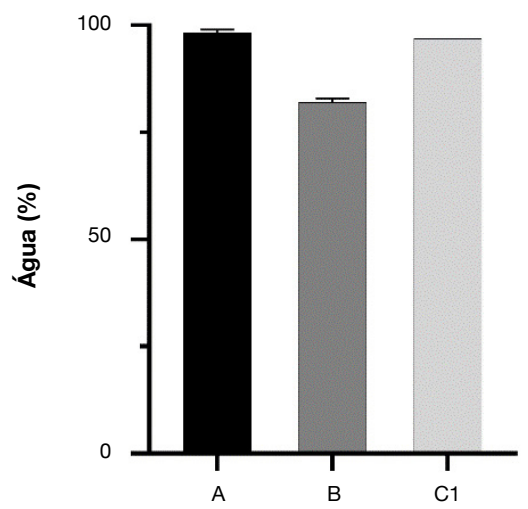

b. Conteúdo de sumo de fruta

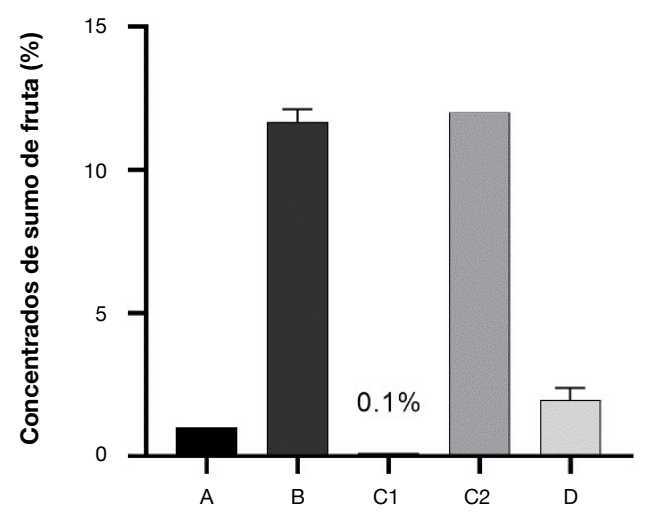

Foram incluídas entre 2 a 5 águas diferentes para cada marca e é apresentada a média e o erro padrão. Para as marcas C2, D e E não foi possível encontrar a percentagem de água presente nos produtos; a marca E não usa sumo de fruta. 
As marcas A, C e E possuem ainda na sua composição conservantes, especificamente o sorbato de potássio (E202), que está presente nos 3 tipos, o benzoato de sódio (E211) - E -, e o dicarbonato dimetílico (E242) - C (Tabela 1). Sendo assim, das várias águas analisadas, as da marca E são as que possuem o maior número de aditivos alimentares, seguidos da $\mathrm{C}$ e da $\mathrm{A}$. No lado oposto, encontram-se as águas da marca B. Por fim, algumas marcas enriquecem as suas águas com outros micronutrientes, nomeadamente magnésio (proveniente de carbonado de magnésio) e cálcio (proveniente de lactato de cálcio) ou algumas vitaminas do complexo B, especificamente a B3, B5, B6, B8, B9 e B12. Esta diversidade nos ingredientes utilizados em cada uma das águas vai naturalmente ter impacto na sua composição nutricional. As principais diferenças são ao nível do teor de hidratos de carbono, nomeadamente açúcares, conteúdo de sal e calorias (Figura 2). Fazendo a comparação por $100 \mathrm{~mL}$ de produto, observa-se que as águas da marca $\mathrm{A}$ e $\mathrm{E}$ são as que possuem uma menor quantidade de hidratos de carbono, inferior a 0,5 g, o que resulta num teor energético muito baixo, inferior a $4 \mathrm{Kcal}$. Em termos de sal, e contrariamente às da marca $\mathrm{E}$, as águas A têm um teor significativo, sendo esta na ordem dos 0,15 g. Os baixos níveis de hidratos de carbono e calorias são consequência da baixa percentagem de sumos de fruta usada combinada com a utilização de adoçantes não-nutritivos. Relativamente às águas das marcas C2 e D, o seu nível de hidratos de carbono é aproximadamente de $5 \mathrm{~g}$, sendo $4 \mathrm{~g}$ correspondentes a açúcares, o que se traduz num teor energético de aproximadamente $20 \mathrm{Kcal}$. É importante salientar que hidratos de carbono têm origens diferentes: no caso das águas C2 provêm de concentrados de fruta, enquanto $D$ resulta do uso de edulcorantes nutritivos. Para estes dois casos o teor de sal é de cerca de 0,1 g. Já as águas $\mathrm{C} 1$ possuem um teor de hidratos de carbono entre 2 e $3 \mathrm{~g}$, e o de açúcares entre 1,2 e $3 \mathrm{~g}$, traduzindo-se num valor energético entre 11 e 16 Kcal. Estas águas não possuem sal. Por fim, de todas as águas analisadas, as águas B são as que possuem uma maior quantidade de hidratos de carbono, entre 4,7 e 6,2 g, e consequentemente de açúcares, entre 4 e 5,6 g. São, por isso, também das mais calóricas: entre 19 e $26 \mathrm{Kcal}$. Importante relembrar que estes hidratos de carbono provêm exclusivamente de sumos concentrado. Estas águas possuem teores mínimos de sal, abaixo de 0,03 g. Nenhuma das águas analisadas possui lípidos e o teor proteico, quando presente, é negligenciável.

\section{DISCUSSÃO DOS RESULTADOS}

É inequívoco o impacto que a alimentação tem ao nível das doenças crónicas não transmissíveis, incluindo o baixo consumo de frutas, hortícolas e fibras, ou o consumo elevado de carnes vermelhas e processadas e de bebidas açucaradas (9). Neste sentido, nos últimos anos, Portugal tem investido na implementação de políticas com o objetivo de promover uma alimentação saudável, incluindo a redução de sal no pão ou o imposto especial de bebidas açucaradas. Dados relativos ao consumo de bebidas açucaradas, à quantidade de açúcar e ao seu teor energético, revelaram uma redução em todos estes parâmetros entre 2017 e 2013, à exceção das águas aromatizadas. Analisando os litros de bebidas refrescantes não alcoólicas vendidos em Portugal em 2016-2017, comparativamente a 2013-2016, é possível observar um aumento de 15,1\% especificamente na categoria das águas aromatizadas. Este foi o único produto deste

\section{Tabela 1}

Classes de aditivos alimentares presentes nas águas com sabor analisadas em cada marca

\begin{tabular}{|c|c|c|c|c|c|}
\hline \multirow{2}{*}{ MARCA } & \multirow{2}{*}{ REGULADOR DE ACIDEZ } & \multicolumn{2}{|c|}{ EDULCORANTES } & \multirow{2}{*}{ CONSERVANTES } & \multirow{2}{*}{ OUTROS } \\
\hline & & NUTRITIVOS & NÃO-NUTRITIVOS & & \\
\hline A & $x$ & & $x$ & $x$ & \\
\hline B & $x$ & & & & \\
\hline C1 & $x$ & $x$ & & $X(2)$ & \\
\hline C2 & $x$ & & & $X(2)$ & \\
\hline D & $x$ & $x$ & & & \\
\hline E & $x$ & & $x$ & $X(2)$ & Maltodextrina \\
\hline
\end{tabular}

Figura 2

Composição nutricional das águas com sabor em função da marca

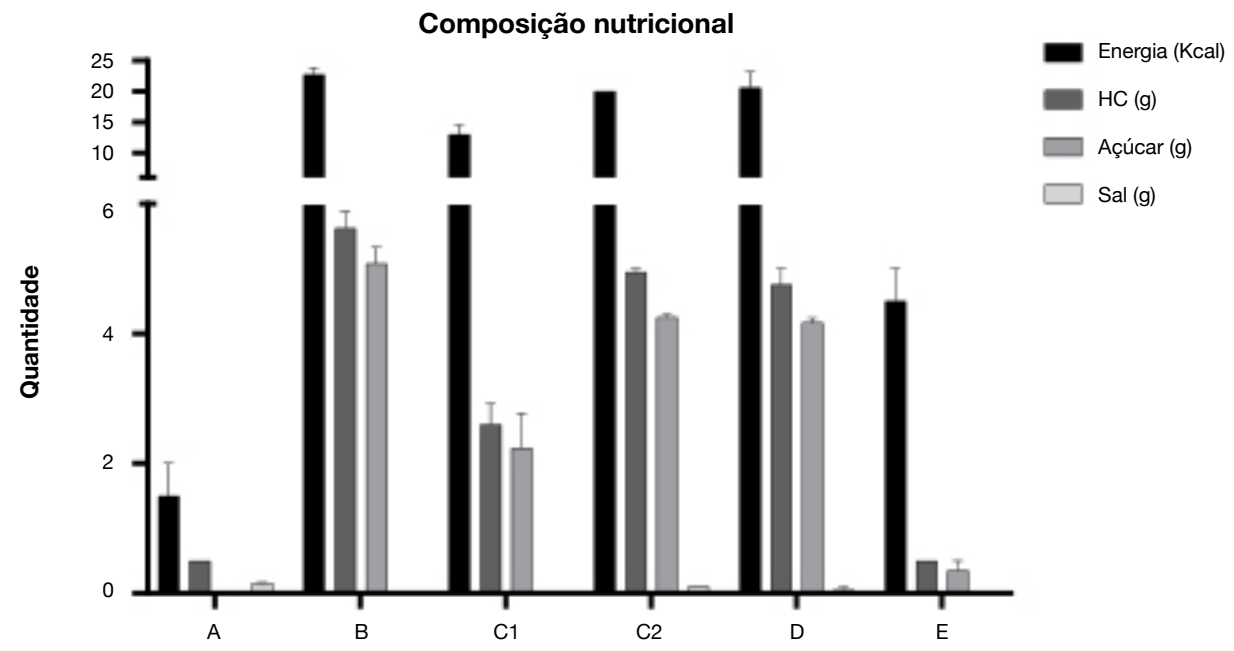

O gráfico mostra os valores de energia (Kcal), hidratos de carbono (HC), açúcares e sal (g) por $100 \mathrm{~mL}$, tendo sido incluídas entre 2 e 5 águas diferentes para cada marca. É apresentada a média e o erro padrão. 
género, no qual se observou um aumento na quantidade de açúcar e, consequentemente, do seu valor energético (10). A variação do seu valor energético entre 2013 e 2017 foi de 116,4\% (11).

Seria importante avaliar as motivações para a ingestão destes produtos em Portugal e que conhecimento têm as pessoas acerca do que efetivamente estão a consumir. Um estudo, realizado em 2010, revelou que o consumo de águas com sabores estava relacionado com a adoção de hábitos alimentares saudáveis, o que poderia sugerir que a perceção geral, em relação a este tipo de alimentos, é/ou se trata efetivamente de um produto saudável (12). O relatório ao Inquérito Alimentar Nacional e de Atividade Física (IAN-AF) de 2015-2016 colocava as águas aromatizadas na categoria "Água" (13).

Os resultados deste trabalho revelam que a grande maioria das águas aromatizadas possui na sua composição edulcorantes. Especificamente no caso dos edulcorantes nutritivos, nomeadamente frutose, vários estudos sugerem que o consumo elevado de bebidas açucaradas com este composto possa estar associado com o aumento na incidência da obesidade e uma redução na sensibilidade à insulina $(14,15)$. Relativamente ao impacto de edulcorantes não nutritivos, vários estudos sistemáticos têm revelado dificuldade em tirar conclusões definitivas em relação aos potenciais benefícios ou riscos do seu consumo (16).

Estudos observacionais parecem mostrar uma associação entre o consumo destes aditivos com um aumento de peso e risco de desenvolvimento de uma série de doenças metabólicas, como a diabetes. Já estudos de intervenção sugerem que a utilização destes aditivos pode ajudar no controlo de peso, principalmente em substituição de adoçantes nutritivos (17). Neste sentido, será importante a realização de ensaios clínicos randomizados que permitam clarificar o impacto de cada edulcorante a nível cardiovascular e metabólico (18). Outro dos riscos associados ao consumo frequente destas águas está relacionado com a erosão dentária, uma vez que alguns dos produtos têm um pH bastante baixo, na ordem dos 3,5 e contêm gás na sua composição (19). Alguma da literatura sugere igualmente que o consumo de bebidas com gás, nomeadamente acima de $300 \mathrm{~mL}$, pode ter um impacto negativo ao nível do sistema gastrointestinal, nomeadamente associação com situações de refluxo gastroesofágico (20). No entanto, esta é ainda uma questão em aberto que requer mais investigação.

A utilização de aditivos alimentares está sujeita a regulamentação por parte da União Europeia, estando definidos os teores máximos autorizados e os alimentos que os podem conter (21). Estão continuamente a ser efetuados estudos no sentido de clarificar potenciais riscos associados ao seu consumo. Por outro lado, é importante ter em conta que vários destes aditivos encontram-se em muitos alimentos diferentes, pelo que pode haver o risco de se ultrapassar os limites máximos recomendados por ingestão combinada de produtos.

\section{CONCLUSÕES}

As águas com sabores, disponíveis em Portugal, são alimentos que apresentam na sua composição vários aditivos alimentares, incluindo edulcorantes e conservantes. Em geral, o seu teor energético e o seu teor de açúcar são consideráveis, contribuindo por isso para um aporte energético aumentado. Algumas águas têm ainda níveis preocupantes de sal. Assim sendo, será importante haver uma maior consciencialização do consumidor face a estes produtos, nomeadamente que se tratam efetivamente de refrigerantes e não de "água", pelo que o seu consumo deve ser reduzido. Por outro lado, alguns dos produtos apresentados têm rotulagem incompleta, sendo essencial que a indústria alimentar forneça ao consumidor todas as informações a nível de ingredientes utilizados.
Seguindo os princípios da "Dieta Mediterrânica" e Roda dos Alimentos portuguesa, o consumo de água é essencial para uma alimentação saudável e deve ser escolhido em relação a qualquer outra opção. Por outro lado, considerando a crescente preocupação com a sustentabilidade da alimentação, deve ser privilegiada a água em natureza, preferencialmente canalizada.

\section{REFERÊNCIAS BIBLIOGRÁFICAS}

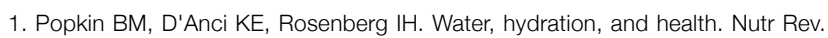
2010;68(8):439-58.

2. Jéquier $E$, Constant $F$. Water as an essential nutrient: the physiological basis of hydration. European Journal of Clinical Nutrition. 2010;64(2):115-23.

3. EFSA Panel on Dietetic Products N, Allergies. Scientific Opinion on Dietary Reference Values for water. EFSA Journal. 2010;8(3):1459.

4. http://www.insa.min-saude.pt/mais-de-96-dos-inquiridos-em-estudo-consomeagua-da-rede-publica-ou-agua-acondicionada/.

5. Maughan RJ, Watson P, Cordery PA, Walsh NP, Oliver SJ, Dolci A, et al. A randomized trial to assess the potential of different beverages to affect hydration status: development of a beverage hydration index. The American Journal of Clinical Nutrition. 2015;103(3):717-23. 6. Cuomo R, Grasso R, Sarnelli G, Capuano G, Nicolai E, Nardone G, et al. Effects of carbonated water on functional dyspepsia and constipation. European Journal of Gastroenterology \& Hepatology. 2002;14(9):991-9.

7. Tiwari BK. Chapter 17 - New Chemical and Biochemical Hurdles. Emerging Technologies for Food Processing (Second Edition). 2014:313-25.

8. Association AD. Position of the American Dietetic Association: Use of Nutritive and Nonnutritive Sweeteners. Journal of the American Dietetic Association. 2004;104(2):255-75. 9. Afshin A, Sur PJ, Fay KA, Cornaby L, Ferrara G, Salama JS, et al. Health effects of dietary risks in 195 countries, 1990\&\#x2013;2017: a systematic analysis for the Global Burden of Disease Study 2017. The Lancet. 2019;393(10184):1958-72.

10. dos Santos CM. Imposto sobre as bebidas não alcoólicas adicionadas de açúucar ou outros edulcorantes: Qual efetividade? Uma análise exploratória. Masters thesis, Universidade Nova de Lisboa. 2018.

11. Baptista S, Silva FGd, Silva D, Landerset F, Simões AL, Gregório MJ, et al. Impacto do Imposto Especial sobre o Consumo de Bebidas Açucaradas e Adicionadas de Edulcorantes: Relatório do Grupo de Trabalho (Despacho N. 2774/2018). FCNAUP - Relatório Técnico. 2018. 12. Ranjit N, Evans MH, Byrd-Williams C, Evans AE, Hoelscher DM. Dietary and activity correlates of sugar-sweetened beverage consumption among adolescents. Pediatrics. 2010;126(4):e754-e61.

13. Lopes CT, D.; Oliveira, A.; Severo, M.; Alarcao, V.; Guiomar, S.; Mota, J.; Teixeira, P.; Rodrigues, S.; Lobato; et al. Inquérito Alimentar Nacional e de Atividade Física (IANAF), 2015-2016 Part 2 Report; University of Porto: Porto, Portugal. 2017.

14. Bray GA, Nielsen SJ, Popkin BM. Consumption of high-fructose corn syrup in beverages may play a role in the epidemic of obesity. The American Journal of Clinical Nutrition. 2004;79(4):537-43.

15. Stanhope KL, Havel PJ. Fructose Consumption: Considerations for Future Research on Its Effects on Adipose Distribution, Lipid Metabolism, and Insulin Sensitivity in Humans. The Journal of Nutrition. 2009;139(6):1236S-41S.

16. Lohner S, Toews I, Meerpohl JJ. Health outcomes of non-nutritive sweeteners: analysis of the research landscape. Nutrition Journal. 2017;16(1):55.

17. Sylvetsky AC, Rother KI. Nonnutritive Sweeteners in Weight Management and Chronic Disease: A Review. Obesity. 2018;26(4):635-40.

18. Khan T, Ayoub-Charette S, Sievenpiper J, Comelli E. Non-Nutritive Sweeteners and Their Effects on Human Health and the Gut Microbiome. 2019.

19. Rees J, Loyn T, Hunter L, Sadaghiani L, Gilmour A. The erosive potential of some flavoured waters. Eur J Dent. 2007;1(1):5-9.

20. Cuomo R, Sarnelli G, Savarese MF, Buyckx. Carbonated beverages and gastrointestinal system: Between myth and reality. Nutr Metab Cardiovasc Dis. 2009 Dec;19(10):683-9.

21. Regulamento (CE) N. ${ }^{\circ}$ 1333/2008 do Parlamento Europeu e do Conselho de 16 de Dezembro de 2008 (relativo aos aditivos alimentares). 